\title{
Determining Robust Reaction Kinetics from Limited Data
}

\author{
Gizem Ozbuyukkaya ${ }^{1}$, Robert Parker ${ }^{1}$, and Goetz Veser ${ }^{1}$ \\ ${ }^{1}$ University of Pittsburgh
}

May 18, 2021

\begin{abstract}
Accurate chemical kinetics are essential for reactor design and operation. However, despite recent advances in "big data" approaches, availability of kinetic data is often limited in industrial practice. Herein, we present a comparative proof-of-concept study for kinetic parameter estimation from limited data. Cross-validation (CV) is implemented to nonlinear least-squares (LS) fitting and evaluated against Markov chain Monte Carlo (MCMC) and genetic algorithm (GA) routines using synthetic data generated from a simple model reaction. As expected, conventional LS is fastest but least accurate in predicting true kinetics. MCMC and GA are effective for larger data sets but tend to overfit to noise for limited data. Cross-validation least-square (LS-CV) strongly outperforms these methods at much reduced computational cost, especially for significant noise. Our findings suggest that implementation of cross-validation with conventional regression provides an efficient approach to kinetic parameter estimation with high accuracy, robustness against noise, and only minimal increase in complexity.
\end{abstract}

\section{Hosted file}

ManuscriptFinal.pdf available at https://authorea.com/users/414317/articles/522371determining-robust-reaction-kinetics-from-limited-data 